\title{
Incisión nasal externa en la corrección de la fisura labial unilateral completa complicada
}

\author{
Dr. Eduardo Egger Zabala; ${ }^{\dagger}$ Dr. Sergin Zúñigat Rocha; ${ }^{1}$ Dr. René Artigas Mambrard; ${ }^{1}$ \\ Dra. Georgina Pelaur Vega;' Sra. Aria María Torres. ${ }^{2}$
}

External nasal incision in the surgical correction of the complete complicated unilateral cleft lip

\begin{abstract}
The present paper is a five years study of the experience in the treatment of nasal lip palate cleft in the Lip and Palate Cleft Clinic from Plastic and Burus Luit of the Servicte of Pediatric Surgerv at Dr. Execuniel Gonzalez. Cortes Childreri's Hospital, with a technigue that pernuts at the age of three month the inmediate cirrection of the nasal area through an external nasal incision joined to labial closure in the complete complicated unilateral cleft lip.

All the patients are evaluated by means st at pre operatory stady and concelusions are taken cout lrom the advantages and disadvantages of this technicue.
\end{abstract}

En Chile, la cirugia del fisurado maso-labior palatino se inicia el año 1920 con el Dr. Agustín Inostroza, de quien se conservan protocolos operatorios de la época, donde se intervenía con la técnica de Mireaux, efectuando las anestesias con elementos como el eter y el clorofurmo.'

Posteriomente, distintos cirujanos como los Dres. Euggenio Díaz Lira; César Izzo Parodi; Arnulfo Johow; Alberto Veloso; Ruthén Acuña y especialnente el Dr. Altredo Gantz Mann, pasan a formar parte de la historia en la evolución alel tratamiento quinirgico del fisurado naso-labio-palatino, utilizando distintas téenicas y obteniendo resultados aceptables para la époc:a.

En 1961 se inicia en el Hospital Matnuel Arriarán la utilización de la Técnica de Ralph Millard, que rápidamente adquiere adeptos, transformándose en la actualidad, en el procedimiento de elección en algunos centros quirúrgicos de nuesto pais.

Los resultados obtenidos a través del tiempo

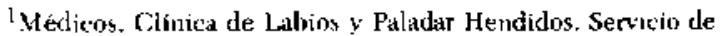
Cinusiat, Flospital E. González Cortés y Departanne nto de Pediatria, Sıb-División Clencias Medicass Sur. Factultad de Medicinu de la U. de Chile:

EEnfenmeta, Servicio de Cinıgía. Hospital E. Conzalez Cortés.
}

con esta técnica nos han permitido valorarla como aceptable si se toma en cuenta la finalidad de reconstruir el labio fisurado, pero, relacionando los conocimientos embriopatológicos de las alteraciones naso-labio-palatinas, y la correspondencia clirecta de la anatomía de la fisura labial con la deforniación nasal, como fuera denıostrada por Avery, permite comprender que el corregir en estos casos, sólo la zona labial, significa sol licionar en torna inconpleta y parcial el problema.

En general, cualesquiera de las técrnicas quiruirgicas utilizadas en la reparación de un labio fisurado, sólo corrige esto último, por tanto, son aplicables cuando se trata de solucionar las fisuras labiales simples, incompletas, no complicadas o complicadas, pero en las cuales sólo existe una fisura alveolar o palatina con contacto de segmentos, y en donde en un $95 \%$ de los casos no existeno son leves las alteraciones nasales.

Cuando se trata de fisuras labio-palatinas completas complicadas, se cumple lo preconizado por Avery, y constihyen un defecto que altera no sólo la zona labial y palatina, sino que tanbién y en un $100 \%$ de los casos, la región nasal, zona en la cual, en general, ninguna técrnica rutirurgica se ha preocupado seriamente, adaptíndose a un criterio de "intocabilidad de la nariz del lactante" y por tan- 
to, dejando dicha zona para ser reparada después de los 16 años de edad, a través de técnicas de rinoplastias convencionales o ${ }^{10,}{ }^{1,4}$ pero permitiendo 10 o más años de vida en los cuales estos pacientes están sometidos a la presión ambiental y escolar, que les asocian trastornos psicológicos de importancia.

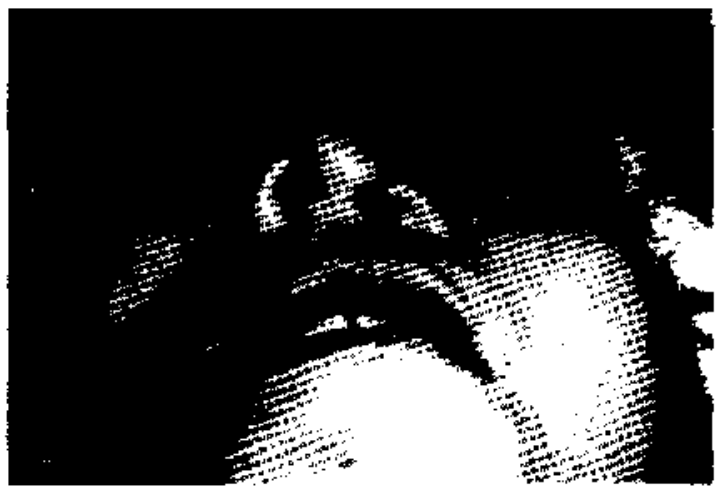

Figura 1. Postoperatorio tardio en L. Leporine Conpleto Complicado (Tec. Ralph-Millard).

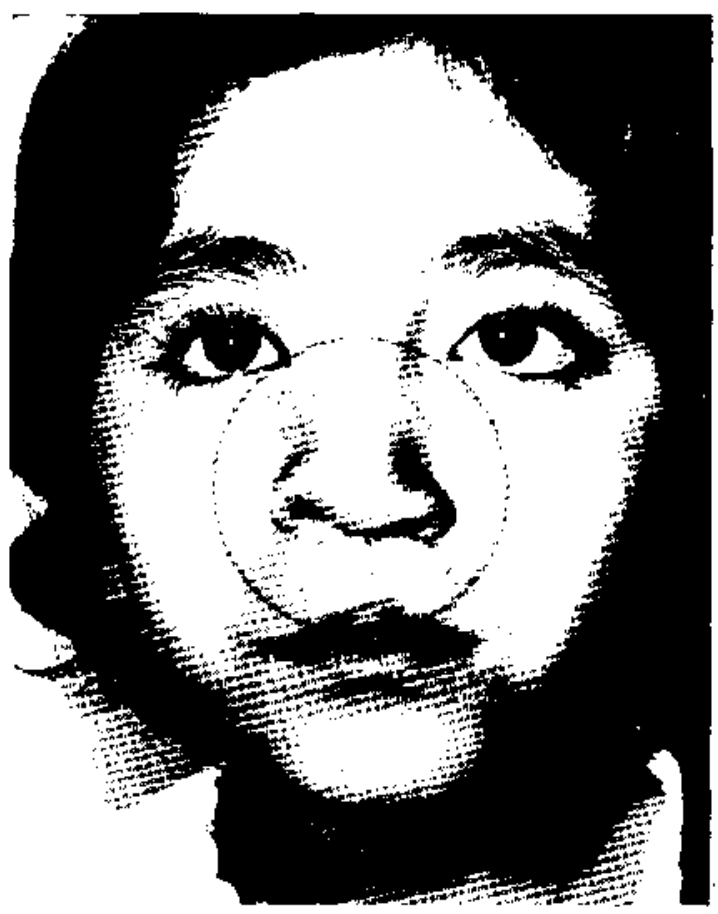

Figura 2. Postoperatorio tardís en Fis. Labial Compl. Complic.

- Cafda punta nasal.

- Disamuonía alar.

En el año 1973 se inicia en el Servicio de Cirugía Pediátrica del Hospital Dr. Exequiel González Cortès, la utilización de la Técnica de Halph
Millard en la corrección de los problemas de los fisurados, dado lo fisiológico de la misma, aunque los resultados generales no fieran los mejores y sólo comparables a los obtenidos con la técnica de Le-Mesurier en boga en esos momentos.

A partir de 1977, y después de la desaparición del Dr. Alfredo Gantz, se constituye la Clínica de Labio y Paladar Hendidos dependiente de la Unidad de Plástica y Quemados del Depto. de Cirugia Pedíátrica del Hospital Dr. Exequiel González Cortés, tomando no solamente en cuenta la parte quirúricica del tratamiento del fisurado, sino, formando un equipo que permite un estudio en conjunto e integral de estos pacientes en su preoperatorio; postoperatorio inmediato $y$ postoperatorio tardío.

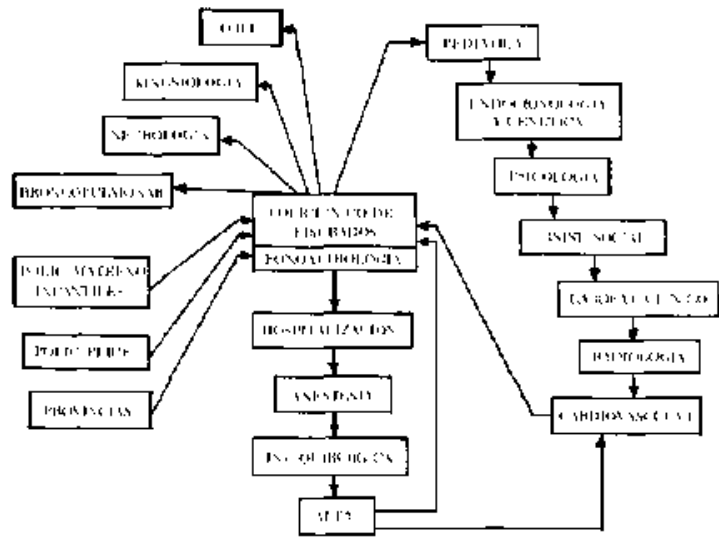

Figura 3. Organigrama Clínica de Labio y Paladar Hendidos.

\section{MATERIAL Y METUDO}

Desde enero de 1977, hasta diciembre de 1981, se intervinieron quirírgicamente 258 pacientes portadores de Fisura Labial Unilateral Completa Complicada, que en definitiva presentan las mayores alteraciones nasales, habiéndose descartado de este total, 21 pacientes intervenidos en la VI, VII y IX región, que por razones de lejaúía y entinentemente económicass, nu pudieron scguir siendo controlados y 33 pacientes que no asistieron a sus controles posteriores, dejando un total de $2(04$ pacientes en este estudio.

En los andisis relacionados con los antecedentes familiatres de los mismos, observamos algunos elementos de importancia que podrian estar incidiendo en el desarrollo de esta patología. Así: 
- En un 31\% no se encontraron antecedentes de importancia.

- En un 25\% de los casos existieron maniobras abortivas en embarazo inicial, preferentemente con la utilización de fármacos.

- En un 20\% existió antecedentes de fisura labial o palatina en padres o parientes.

- En un $15 \%$ se encontró alcoholismo en tunoo ambos padres.

- En el $9 \%$ restante existieron antecedentes de atmenazas de aborto espontáneo; diahetes en padres o parientes; antecedentes de radiografias antes del tercer mes de embarazo y enfenmedad viral (rubéola) en uno de los casos en los prinieros tres meses de la concepción.

Los estudios cardiológricos y broncopulmonares previns a la intervención detectaron un $3,30 \%$ de pacientes con comunicación interventricular permeables al tercer mes de vida y en un $5 \%$ atelectasias pulmonares, elementos éstos coue condicionaron la condacta anestésica para pertuitir mejores posibilidades al paciente y el acto quirirgico.

El $80 \%$ de los casos presentaron brouquitis repetidas que condicionaron la no intervención en el tiempo y momento oportino.

Desde el punto de vista de su estudio genético, se comprobó que en un $20 \%$ existe una predisposición genética familiar.

Otorrinolaringológicamente en el 56\% de los casos se presentaron otitis repetidas.

El estudio social efectuado a estos pacientes nos demostró cue en el 76\% de los casos, el ingreso econónico familiar fue menos de US $\$ 200$, correspondiendo a la mitad de ellos, ingresos aún inferiores a US 100 mensuales.

Un 16\% presentó ingresos entre 200 y 300 dólares al mes, y sólo en un $8 \%$ ingresos superioress a los US $\$ 300$ mensuales.

El estudio y valoración psicológica del paxiente y su medio afectivo en padres y familiares cercanos, nos pemitió entender y valorar la teraṕéutica, dividiéndose los pacientes en cuatro categorías.

Grupo I: Susceptibles de tratamiento integral $y$ maximo de esfuerzos . . . 30\%

Grupo 2: Susceptibles de tratamiento iutegral y ayuda psicológica en padres y parientes $\ldots \ldots \ldots \ldots \ldots \ldots .48 \%$ Ambos grupos representaton el 78 \% de este estudio.

Grupo 3: Susceptibles de tratamientos parciales y/o totales de acuerdo a con- diciones conductuales de padres o parientes ............... 10\%

Grupo 4: Susceptibles de tatamiento de problemas estéticos, físicos $y$ mentales en el menor tiempo posible, incluyendo varios tiempos quiruirgicos en uno solo, dada las pobres condiciones intelectuales (paciente, padres o parientes); geográficas; físicas; nutricionales; de motivatión, etc., ......... 12\%

\section{TECNICA}

La utilización de la Técnica de Ralph-Millard es la base que se ha tomado para canalizar la coпесción nasal concomitante en estos pacientes. $\mathrm{Si}$ bien esta técnicu nos ha dado excelentes resultados en los pacientes portadores de fisuras labiales incompletas no complicudas, no ha ocurrido to mismo en los pacientes con fisuras labiales conpletas complicadas en los que se observa una dislocación del cartílago alar del lado tisurado, que condiciona una caida de la punta nasal, con una disminucion en altura de la hemi-columela afectada que oscila entre un 15 y $20 \%$ en relación al lado sano y un desplazamiento del ala nasal importante. (Fig. 4 y 5).

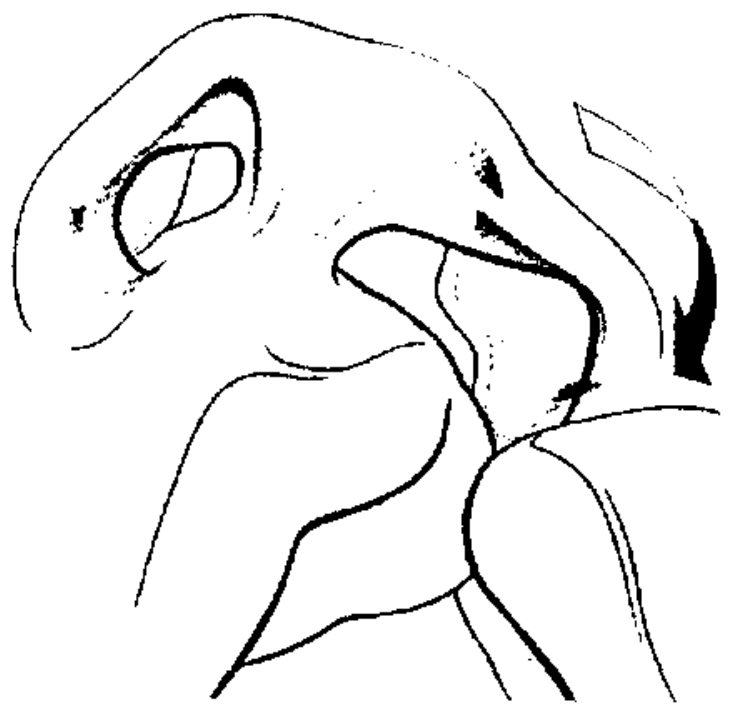

Figura 4.

La utilización de la Técnica de Ralph-Millard no permite la reparación de este problema, razón por la cual utilizamos, siguiendo la Escuela Mexi- 


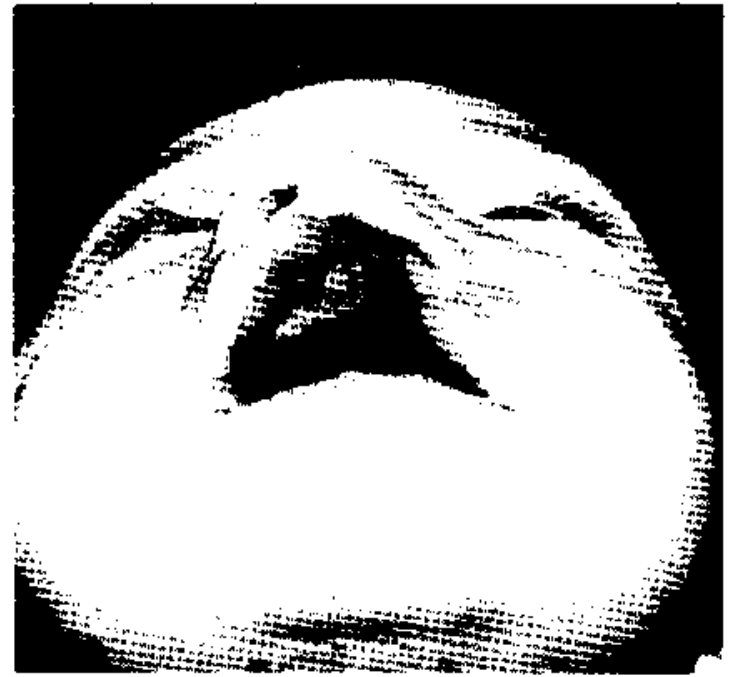

Figura 5.

cana del Dr. Fernando Ortiz Monasterio, ${ }^{12,13 ; 14: 15}$ 16. ${ }^{17}$ la incisión nasal externa, lo que nos pentite llevar y unir en su cúpula el cartilago alar afectatdo, reconstituyendo por tanto, lo más fisiológicamente posible lo que está alterado y utilizando el colgajo " $\mathrm{C}$ " de Millard en la reconstitución de un buen piso nasal. (Fig. 6.)

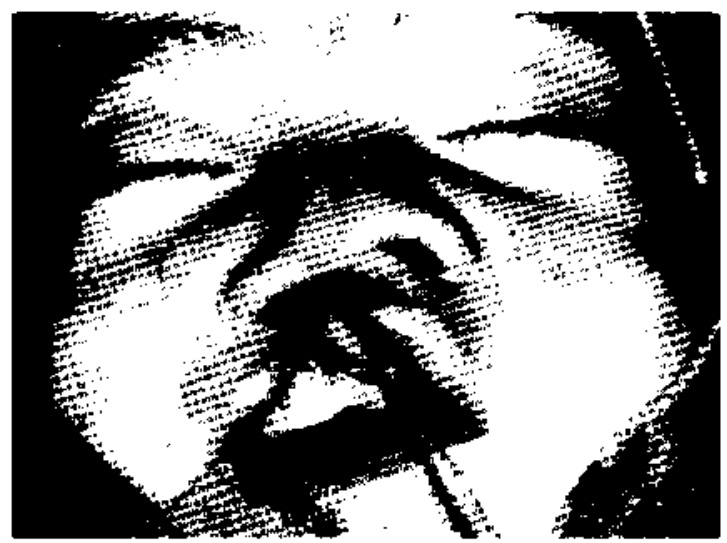

Figura 6. RALPH-MILLARD más incisión nasal externa.

El utilizar la incisión nasal externa condiciona además el tener que resecar un exceso de piel nasal que será variable entre algunos milímetros hasta un y medio centímetro, dependiendo del grado de dislocación de ese cartílago alar.

A pesar de corregir la punta nasal, en atgmnos casos se produce la caída del cartílago alar en la zona medial del arco nasal reparado, razón por la cual y para evitar dicho problema, colocanos un

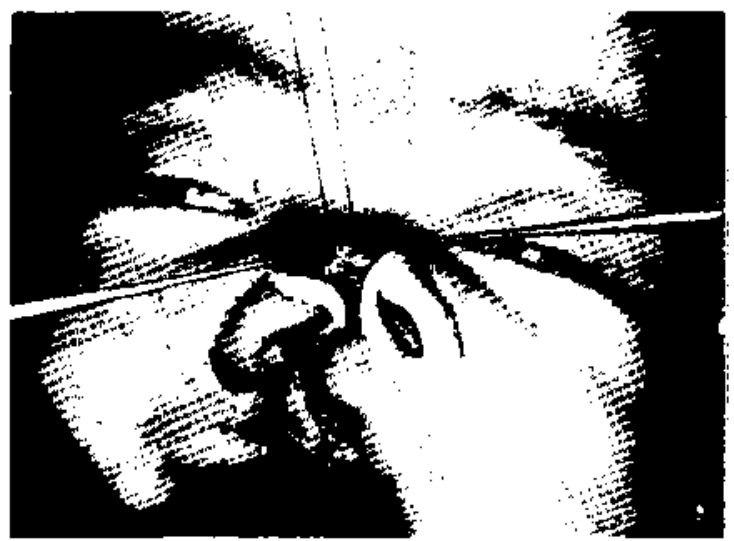

Figura 7. Unión en túpula de cartílago alar afectado.

pun to de hilo nonofilamento uniendo dicha zona a los cartílagos nasales laterales, solucionando en forta definitiva dicha complicación postoperatoria tardia.

El cierre labial se efectía de acuerdo a la técnica de Ralph-Millard, teniendo especial cuidado en la reconstrucción muscular y de la inucosa, a fin de construir un buen vestíbulo labial y evitur las adherencias a ese nivel, situación corrientemente observada cuando es mal empleada esta técnica (Fig. 8).

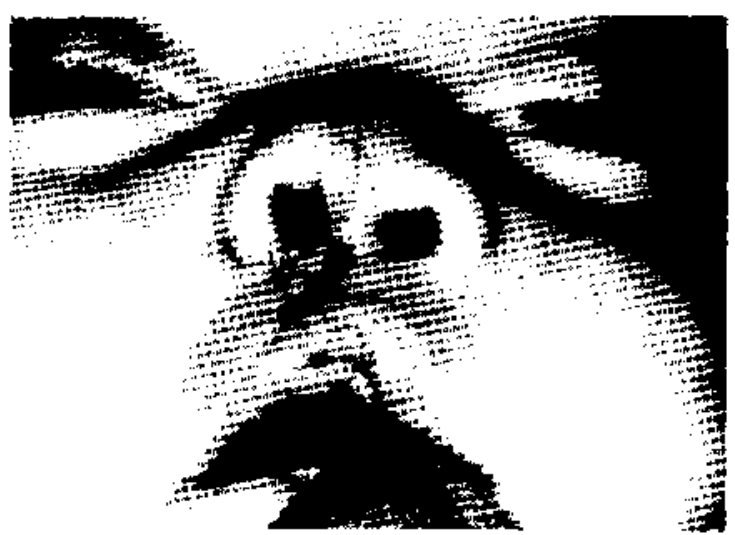

Figura 8. Postoperatorio inmediato con sobrecorrección alar.

Se ha sustituido y en forma definitiva la utilización de elementos de sutura como la seda por su condición de trenzada, auncjue revestida de peliculas imperneables, es fácil presa de los elementos tisulares que provocan su maceración y la penetración de gérmenes que condicionan infección y ser la causa de las dehiscencias tan comunes de observar; por esto, preferimos los hilus monofíla- 
mentos, con los cuales se ham evitado en un $99,5 \%$ dichas complicaciones.

Se ha eliminado en forna absoluta la limpieza de la zona operada mediante la aplicación de cremas, ya que éstas formian una pelicula sobre la zona interven ida, constituyendo costras fácilmente infectantes por la maceración de la piel: preferimos por tanto, el simple aseo con suero tisiológico cuando las condiciones asi lo indican.

Se utilizan soluciones de adrendina o noradrenalina como infiltración intraoperatoria. Los puntos son retirados al cuarto día en la región nasal y al sexto o séptimo de la región labial. La alimentación se reanuda con líquidos y cuchara a las dos horas de la intervención, manteniéndose así hasta las 18 horas de postoperatorio, para continuar con papillas durante la primera semana.

\section{RESULTADOS}

Siendo un acto quirúrgico realizado en un lactante y a los tres meses de edad, las complicaciones obtenidas y valoradas en el postoperatorio inmediato se refirieron a:

- $1 \%$ cuadros nemuónicos.

$-0,5 \%$ de dehiscencias postinfecoión.

Ias complicaciones postoperatorias tardias se refirieron especificantente a:

- $1 \%$ caída de la regiós alar.

- 6\% caída de zona media alar.

$-0,5 \%$ obstrucción nasal parcial.

La valoración de los resultados estético-fincionales en los cuales se tomaron en cuenta la invisibilidad progresiva de la incisión del dorso nasal; la permeabilidad de los conductos naskles y lit conformidad o node los padreso parientes, fineron citalogados en:

- Malos .................... $1.5 \%$

- hegulares $\ldots \ldots \ldots \ldots \ldots \ldots \ldots \ldots 6,5 \%$

- Buenos ...................... $92 \%$

En la evolución de los pacientes intervenidos se comprobó lo siguiente:

- Retardo del crecimiento nasal ..... $0,0 \%$

- Conformación estético-funcional normal ..................... $92,0 \%$

— Cicatriz queloide orificio nasal .... $1,0 \%$

- Obstrncción nasal parcial ........ $0,5 \%$

- Caída total o parcial del ala nasal . . $\quad 6,5 \%$

La técrica utilizada condiciona interrogantes que la experiencia acumulada nos permiten contestar a la luz de los resultados, que la incisión nasal no provoca alteraciones del crecimiento na-
INCISIOY NASAL FXTEHA

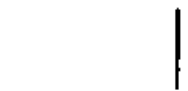

INITSIHILIDAD A PAHTIR

DEL SET, MES POSTOPEH.

Figura 9.

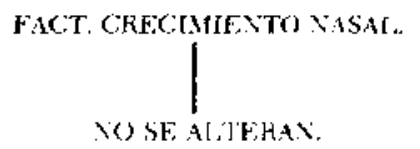

Figura 10.

sal y la incisión nasal misma comienza a desaparecer desde el tercer mes de postoperatorio, provocando menos tastomos cicatrizales que la propia incisiôn labial (Fig. 11 a 15 ).

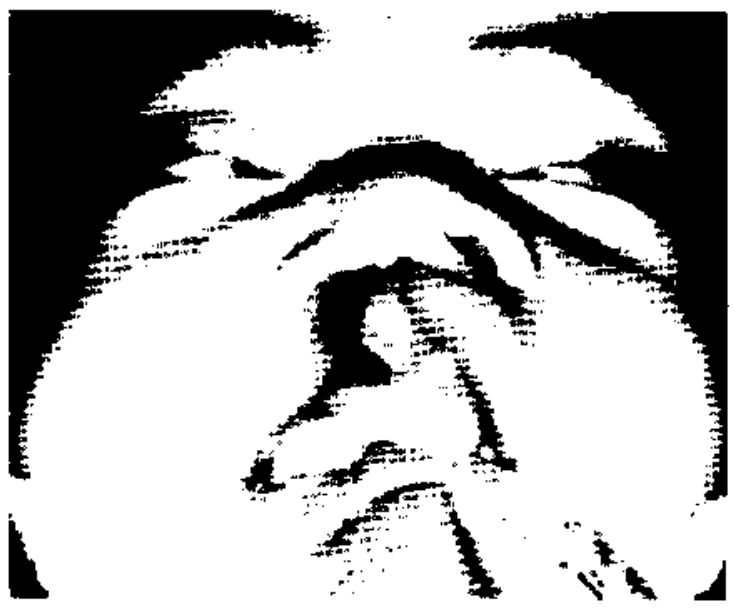

Figura 11. Preoperatorio.

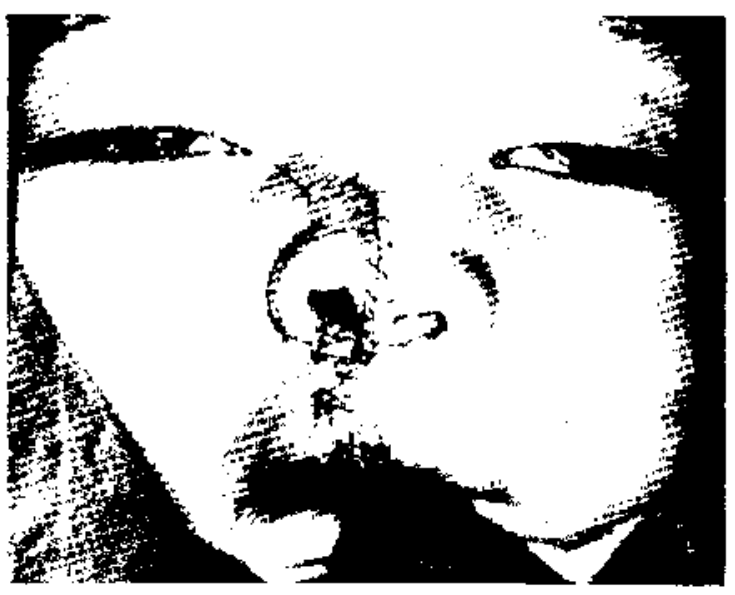

Figura 12. Postoperatorio ínmediato (sobreconección nasal!. 


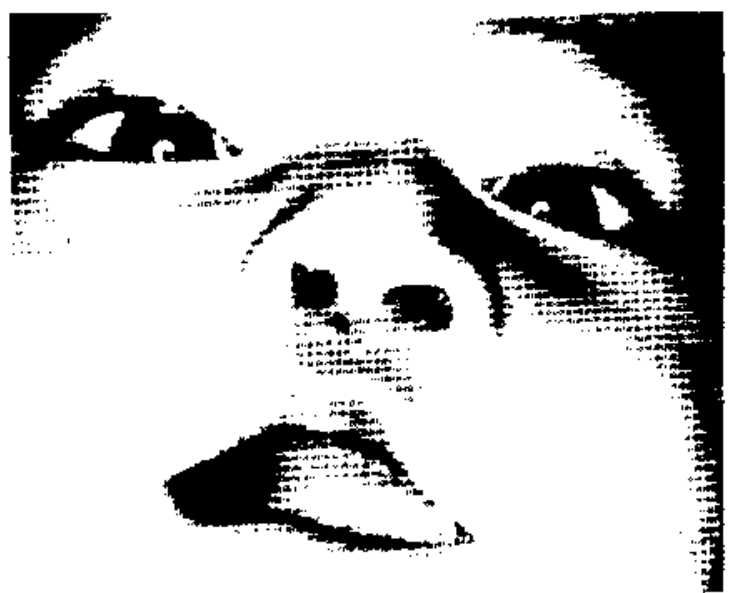

Figura 13. Postoperatorio 6 meses. Hemicolumela a nivel. Arco alar conservado. Inc: nasal prícticamente invisible.

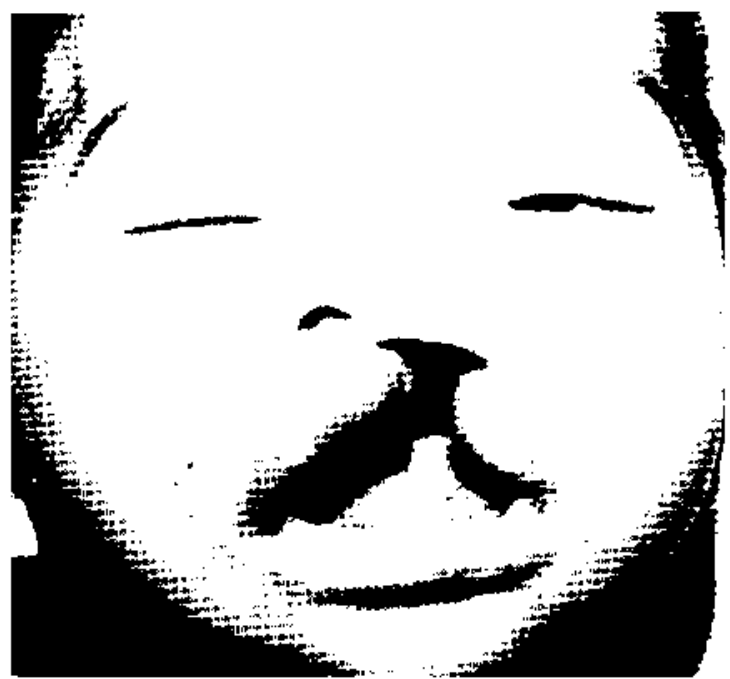

Fjgura 14. Preoperatorio.

\section{RESUMEN}

A través del presente trabajo se hace un estudio de cinco años de experiencia en el tratamiento del Fisurado naso-labio-palatino en la Clínica de Labio y Paladar Hendidos dependiente de la Unidad de Cirugía Plástica y Quemados del Dpto. de Cirugía Pediatrica del Hospital Dr. Exequiel González Cortés, con una técnica que permite, a los tres meses de edad, efectuar concomitantemente al cierre labial con la Técnica de Ralph Millard, la corrección inmediata de la zona nasal a través de una incisión nasal externa, considerando cue esta zoma esta profundamente alterada en las fisturas

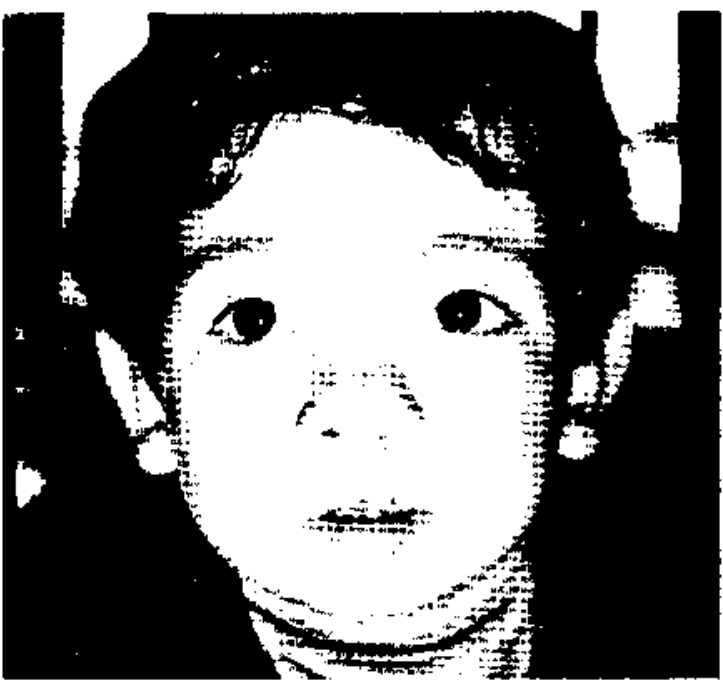

Figura 15. 4 años 6 meses postoperatorio. Punta nasal conservada. Arco y nivel alar conservado. Inc. nasal apenas visible.

labiales unilaterales completas complicadas.

Se valoran los pacientes por medio de un estudio preoperatorio integral y se extraen las conclusiones de los problemas y ventajas que esta tecnical otorgat.

\section{REFERENCLAS}

1 Anuario clel Hospital Manuel Ariarán. 1921.

2 Anery, J.K. The nasal capsule in cleft palata. Anat. Aız. (Suppl); $109 ; 1962$

${ }^{3}$ Conterse, $/$ M. Reconstructive Plastic Suryery. Vol. 4; Second Editiot, 1977 .

${ }^{4}$ Equer Zavola. E. Fisura libial unilateral cmonpleta compticada: cierre labial y comección nasal inmediata. Revista Sch: Cinjinos de (hile. Trabajo de lugreso. (En prensa). 1982.

SGantz Mann, A. Nuevos conceptos en el trataruieuts de la Fisura Palatina. Imp. y Librerie Leblanc. 1943.

"Gantz Mann, 4. Tratamiento del Labio Leporino y lit Fisurd Palatina. Tesis U. rle Chile. 1968.

Tohow. A. Labio Leporina y r isura Yalatina. Tesis U. de Clule. 1938.

'Cleft Lip and Palate technitule. St. Thonus Homp. Rep. 2; 127 ; 1934.

Millard. R. D. Clett Gratt. Tamo I. The unilateral deformities 1976.

10 Millard, H. D. Cleft Graft. Tomo III. Alveolar and palatal deformities. 1980.

IJODonnel, $\mathrm{J}$. An antalysis of presurgical orthopedics in the treatunent of unilateral lip and palate. The Cleft Palate Joumal. Vol. 11; 374; Oct. 1974 .

12 Ortiz Monasterio, F; Serrano, A. A sndy of untreated adult clett palate patients. Plat. and Rec. Surgery; 34; 36; 1966.

13 Ortiz Monasterio, $F_{\text {, }}$ Sertano, A. Cefalometric measurements un adult patients with nom-opereated cleft palates. Plast. and Hec. Surgery. 24; $53 ; 1979$.

${ }^{4}$ Ortiz Monasterio, F.; Olmedo, A,; Trigos, 1. Final results from 
the delayed treatinent of patients with clefts of the lip and palate. Scand. J. Plast. Rec. Sury. N. ${ }^{\circ}$ : 108: 1974.

${ }^{15}$ Trizos, 1.; Garcra, C.; Ortiz Monasterio, F. Tratamiento integral temprano del paladar primario hendido. Cir. Plast. lberolatimoanericana Vol. $5 ; 2 \overline{7} ; 1979$.

${ }^{16}$ Trigos, $I$; Alonso, P.R. Queilonasoplastia primaria mediante la técuica del colgajo trianguliur modificado. Cir. Plast. Ibero- latinoasuericana. Vol. Vl; N. ${ }^{0} 3 ; 297 ; 1979$

${ }^{13}$ Velasquez, M.; Ortiz Monasterio, F. Primary simultanerus correction of the lip and uose in the unilateral cleft lip. Plat. Rec. Surg. 54: 25I; 1974

I8Vitar Sancha, B. Labio leporino unilateral. Valoración global de los resultados obtenidos a largo plizo. Cir. Plast Iberolattomanericana. N. ${ }^{0}$; 23 ; 1975. 